$\mathrm{DTP} / 95 / 82$

December 1995

\title{
Polarized Parton Distributions in the Nucleon
}

\author{
T. Gehrmann and W.J. Stirling \\ Departments of Physics and Mathematical Sciences, University of Durham \\ Durham DH1 3LE, England
}

\begin{abstract}
The distribution of the spin of the nucleon among its constituents can be parametrized in the form of polarized parton distribution functions for quarks and gluons. Using all available data on the polarized structure function $g_{1}\left(x, Q^{2}\right)$, we determine these distributions both at leading and next-to-leading order in perturbation theory. We suggest three different, equally possible scenarios for the polarized gluon distribution, which is found to be only loosely constrained by current experimental data. We examine various possibilities of measuring polarized parton distributions at future experiments.
\end{abstract}




\section{Introduction}

Understanding the spin structure of the nucleon is one of the most important questions in strong interaction physics today. There has been renewed interest in recent years, largely as a result of a series of high-precision polarized deep inelastic scattering experiments. So far, the bulk of the information comes from measurements of the spin-dependent structure function $g_{1}\left(x, Q^{2}\right)$. From such measurements, spin-dependent parton distributions can be extracted and compared with theoretical models, or used to make predictions for future experiments. Several theoretical issues have received particular attention: the predictions of various sum-rules and the measurement of $\alpha_{s}$, the magnitude of the polarized gluon distribution, and the behaviour of the polarized parton distributions at small $x$.

In a previous study [1] we performed a global analysis of all available deep inelastic polarized structure function data in the context of the QCD-improved parton model at leading order. We supplemented the experimental information with theoretical assumptions about the flavour content and the form of the distributions at large and small $x$. In particular, we presented three different sets of parton distributions characterized by qualitiatively different polarized gluon distributions, and suggested further experiments which could discriminate between them.

Since the work of Ref. [1] there have been several important advances. More precise polarized structure function data has become available. Both the the SMC [2] and SLAC-E143 collaborations [3] have measured the structure function $g_{1}$ of the deuteron, supplementing their previous proton target measurements, and the SLAC-E143 collab-

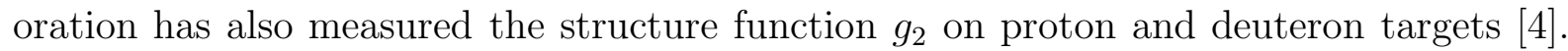
The new $g_{1}$ data are particularly important for determining the polarized $u$ - and $d-$-quark distributions separately.

A second advance has been the theoretical calculation [5] of the next-to-leading order contributions to the polarized splitting functions $\Delta P_{i j}\left(x, \alpha_{s}\right)$ which determine the $Q^{2}$ evolution of the distributions. This not only improves the precision of the phenomenology, but also allows for the first time a consistent factorization/renormalization scheme dependence to be imposed on the analysis.

In this study we repeat the analysis of [1] using the new polarized structure function data, but now at both leading and next-to-leading order in perturbation theory. Our aim, as before, is to derive a consistent set of polarized parton distributions. At the same time we can check that the $Q^{2}$ dependence seen in the data is consistent with the predictions of perturbative QCD. We discuss in detail the constraining power of the various data sets, the role of the polarized gluon distribution, and the prospects for future measurements. A similar analysis of polarized structure functions has been reported recently in Ref. [6], using the 'dynamical parton model' approach in which the distributions at small $x$ are generated dynamically from valence-like distributions at a small starting $Q_{0}^{2}$ scale. A reasonable description of the data is obtained. Our approach, in contrast, is to constrain 
the small $x$ distributions from the data, without prejudice as to the origin of the observed behaviour. This is in the same spirit as the unpolarized analysis of Refs. [7, 8].

We begin by reviewing the basic theoretical input to the analysis. The fundamental quantity of interest is the structure function $g_{1}$ which, in analogy with the unpolarized structure function $F_{1}$, can (in the QCD-improved parton model at leading order) be expressed in terms of the probability distributions for finding quarks with spin parallel or antiparallel to the longitudinally polarized parent nucleon:

$$
\begin{aligned}
F_{1}\left(x, Q^{2}\right) & =\frac{1}{2} \sum_{q} e_{q}^{2}\left[q\left(x, Q^{2}\right)+\bar{q}\left(x, Q^{2}\right)\right] \\
g_{1}\left(x, Q^{2}\right) & =\frac{1}{2} \sum_{q} e_{q}^{2}\left[\Delta q\left(x, Q^{2}\right)+\Delta \bar{q}\left(x, Q^{2}\right)\right],
\end{aligned}
$$

where

$$
q=q_{\uparrow}+q_{\downarrow}, \quad \Delta q=q_{\uparrow}-q_{\downarrow} .
$$

The first moment of $g_{1}\left(x, Q^{2}\right)$ measures the expectation value of the axial-vector current between two identical nucleon states:

$$
\Gamma_{1}^{p, n}= \pm \frac{1}{12} a_{3}+\frac{1}{36} a_{8}+\frac{1}{9} a_{0}
$$

where the conserved nonsinglet axial vector currents are known from the hyperon decay constants (the Ellis-Jaffe sum rule [9, 10]):

$$
\begin{aligned}
& a_{3}=F+D \\
& a_{8}=3 F-D .
\end{aligned}
$$

In contrast, the singlet axial vector current $a_{0}$ is non-conserved due to the axial anomaly. It cannot be related to any other experimental quantity, although several model-dependent estimates of it can be found in the literature: if the total nucleon spin is carried only by quarks one finds $a_{0}=1$ [9], and if the strange quark sea in the nucleon is unpolarized then $a_{0}=a_{8} \approx 0.579$ [10]. Both these estimates are clearly ruled out by experimental measurements [2, 3, 11], which find $a_{0} \approx 0.15-0.3$ in the range $3 \mathrm{GeV}^{2}<Q^{2}<12 \mathrm{GeV}^{2}$.

In the QCD-improved parton model $g_{1}$ is expressed in terms of parton distributions for the polarization of quarks and gluons,

$$
\begin{aligned}
g_{1}\left(x, Q^{2}\right)= & \frac{1}{2} \sum_{q} e_{q}^{2} \int_{x}^{1} \frac{d y}{y}\left[\Delta q\left(x / y, Q^{2}\right)+\Delta \bar{q}\left(x / y, Q^{2}\right)\right] \\
& \times\left\{\delta(1-y)+\frac{\alpha_{s}\left(Q^{2}\right)}{2 \pi} \Delta C_{q}(y)+\ldots\right\} \\
& +\frac{\left\langle e_{q}^{2}\right\rangle}{2} \int_{x}^{1} \frac{d y}{y} \Delta G\left(x / y, Q^{2}\right)\left\{n_{f} \frac{\alpha_{s}\left(Q^{2}\right)}{2 \pi} \Delta C_{G}(y)+\ldots\right\} .
\end{aligned}
$$


The variation of these polarized parton distributions with $Q^{2}$ is determined by the GLAP evolution equation [12]:

$$
\begin{aligned}
& \frac{\partial}{\partial \ln Q^{2}}\left(\begin{array}{c}
\Delta q \\
\Delta G
\end{array}\right)\left(x, Q^{2}\right) \\
& \quad=\frac{\alpha_{s}\left(Q^{2}\right)}{2 \pi} \int_{x}^{1} \frac{\mathrm{d} y}{y}\left(\begin{array}{cc}
\Delta P_{q q} & \Delta P_{q G} \\
\sum_{q} \Delta P_{G q} & \Delta P_{G G}
\end{array}\right)(y)\left(\begin{array}{c}
\Delta q \\
\Delta G
\end{array}\right)\left(x / y, Q^{2}\right) .
\end{aligned}
$$

For a consistent theoretical description of $g_{1}\left(x, Q^{2}\right)$, the coefficient functions in (66) must be truncated at the same order in perturbation theory as the splitting functions in (7). Although the $\mathcal{O}\left(\alpha_{s}\right)$ corrections to the coefficient functions $\Delta C_{q}(y)$ [13] and $\Delta C_{G}(y)$ [14] have been known for a long time, a next-to-leading order study was not possible until very recently, as the $\mathcal{O}\left(\alpha_{s}\right)$ corrections to the $\Delta P_{i j}(y)$ were not known. These have now been published for the first time in Ref. [5].

Using the next-to-leading order evolution equations [15], the unpolarized parton distributions can be determined to a high level of accuracy (see for example Refs. [7, 8, 16]) using a wide variety of high-precision experimental data from different processes. As the only data available on the spin structure of the nucleon are the structure function measurements of Refs. [2, 3, 11], we are still far from obtaining a similar level of precision on the polarized distributions.

The appropriate choice of scheme for $g_{1}$ in the QCD-improved parton model has been a matter of discussion over the last few years (see for example [17] for a recent review). The main issue is the treatment of the gluonic contributions to the Ellis-Jaffe sum rule. While the nonsinglet contributions to this sum rule can be related to conserved currents and are unambiguously defined, there is an arbitrariness in the decomposition of the singlet contribution [14, 18], depending on the absorption procedure used for the axial anomaly. In the $\overline{\mathrm{MS}}$ scheme, this anomaly is absorbed into the next-to-leading order pure singlet quark-to-quark splitting function [19]. Another possible procedure is to allow for an anomalous gluonic contribution to the Ellis-Jaffe sum rule, which is absent in the $\overline{\mathrm{MS}}$-scheme. The appropriate scheme transformation [19] then yields conservation of the quark contribution to $a_{0}$. In the present study we will work entirely in the $\overline{\mathrm{MS}}$ scheme. Any other choice of scheme would tie the resulting distributions to $g_{1}$, as higher-order corrections to the coefficient functions of other processes are so far only known in the $\overline{\mathrm{MS}}$ scheme.1

The choice of the $\overline{\mathrm{MS}}$ scheme also allows us to use our distributions in conjunction with a broad range of unpolarized distributions, the majority of which are defined in this

\footnotetext{
${ }^{1} \mathrm{~A}$ NLO analysis of polarized deep inelastic scattering data in the so-called Adler-Bardeen scheme, which displays different factorization properties, has recently been carried out [20]. In Ref. [20] the emphasis is on the determination of the singlet axial charge $a_{0}$ and the first moment of the polarized gluon distribution.
} 
scheme. For reference, the $\overline{\mathrm{MS}}$ scheme coefficient functions in (的) are

$$
\begin{aligned}
\Delta C_{q}(y)= & C_{F}\left\{2\left(\frac{\ln (1-y)}{1-y}\right)_{+}-\frac{3}{2}\left(\frac{1}{1-y}\right)_{+}-(1+y) \ln (1-y)\right. \\
& \left.-\frac{1+y^{2}}{1-y} \ln y+2+y+\delta(1-y)\left(-2 \zeta(2)-\frac{9}{2}\right)\right\} \\
\Delta C_{G}(y)= & T_{R}\left\{2(2 y-1) \ln \left(\frac{1-y}{y}\right)+2(3-4 y)\right\}
\end{aligned}
$$

In the following section we will present the results of our leading and next-to-leading order parton model analyses of polarized structure function data. Section 3 discusses the prospects of various future experiments on polarized nucleons and Section 4 contains a brief summary and our conclusions.

\section{Polarized parton distributions in leading and next- to-leading order}

We adopt a similar approach to the global analysis of unpolarized parton distributions in the nucleon [7] by parametrizing the polarized distributions at the starting scale in the form:

$$
\begin{aligned}
x \Delta u_{v}\left(x, Q_{0}^{2}\right) & =\eta_{u} A_{u} x^{a_{u}}(1-x)^{b_{u}}\left(1+\gamma_{u} x+\rho_{u} x^{1 / 2}\right) \\
x \Delta d_{v}\left(x, Q_{0}^{2}\right) & =\eta_{d} A_{d} x^{a_{d}}(1-x)^{b_{d}}\left(1+\gamma_{d} x+\rho_{d} x^{1 / 2}\right) \\
x \Delta \bar{q}\left(x, Q_{0}^{2}\right) & =\eta_{\bar{q}} A_{\bar{q}} x^{a_{\bar{q}}}(1-x)^{b_{\bar{q}}}\left(1+\gamma_{\bar{q}} x+\rho_{\bar{q}} x^{1 / 2}\right) \\
x \Delta G\left(x, Q_{0}^{2}\right) & =\eta_{G} A_{G} x^{a_{G}}(1-x)^{b_{G}}\left(1+\gamma_{G} x+\rho_{G} x^{1 / 2}\right),
\end{aligned}
$$

where we take $Q_{0}^{2}=4 \mathrm{GeV}^{2}$. The normalization factors are

$$
A_{f}^{-1}(f=q, G)=\left(1+\gamma_{f} \frac{a_{f}}{a_{f}+b_{f}+1}\right) \frac{\Gamma\left(a_{f}\right) \Gamma\left(b_{f}+1\right)}{\Gamma\left(a_{f}+b_{f}+1\right)}+\rho_{f} \frac{\Gamma\left(a_{f}+0.5\right) \Gamma\left(b_{f}+1\right)}{\Gamma\left(a_{f}+b_{f}+1.5\right)}
$$

which ensures that the first moments of the distributions, $\int_{0}^{1} d x \Delta f\left(x, Q_{0}^{2}\right)$, are given by $\eta_{f}$.

Various experimental measurements of unpolarized lepton $(=e, \mu, \nu)$-nucleon and unpolarized Drell-Yan cross sections yield a reasonably precise flavour decomposition of the light quark $(u, d, s)$ sea. Such a decomposition is not yet possible for the polarization of the light quark sea, as measurements of the structure function $g_{1}$ are only sensitive to the charge weighted sum of all quark flavours, not to the individual distributions. We therefore assume a $\mathrm{SU}(3)$-symmetric antiquark polarization $\Delta \bar{q}\left(x, Q_{0}^{2}\right)=\Delta \bar{u}\left(x, Q_{0}^{2}\right)=$ 
$\Delta \bar{d}\left(x, Q_{0}^{2}\right)=\Delta \bar{s}\left(x, Q_{0}^{2}\right)$. This ad-hoc assumption is only justified at the present level of experimental knowledge, and is furthermore immediately broken by next-to-leading order evolution [21].

The first moments of the polarized quark distributions can be determined from the measured values of the Ellis-Jaffe sum rule. Imposing SU(3)-symmetry at $Q_{0}^{2}$, this sum rule reads

$$
\Gamma_{1}^{p, n}\left(Q_{0}^{2}\right)=\left(1-\frac{\alpha_{s}\left(Q_{0}^{2}\right)}{\pi}\right)\left( \pm \frac{1}{12} a_{3}+\frac{1}{36} a_{8}+\frac{1}{9} a_{0}\left(Q_{0}^{2}\right)\right)
$$

with

$$
\begin{aligned}
a_{3} & =\eta_{u}-\eta_{d}=F+D, \\
a_{8} & =\eta_{u}+\eta_{d}=3 F-D, \\
a_{0}\left(Q_{0}^{2}\right) & =\eta_{u}+\eta_{d}+6 \eta_{\bar{q}}\left(Q_{0}^{2}\right) .
\end{aligned}
$$

In this approach, the first moments of the valence quark polarizations are obtained from the nonsinglet axial-vector current matrix elements [22], while the first moment of the sea quark distribution is inferred from the measured value of $\Gamma_{1}$. For the leading-order (LO) distributions, we correct the normalization of $\eta_{u}$ and $\eta_{d}$ by the $\mathcal{O}\left(\alpha_{s}\right)$ coefficient function in (11]) [1]. The first moments obtained by this procedure are listed in Table [1. Note that the Ellis-Jaffe sum rule is a $\left(Q^{2}\right.$-independent) constant at leading order in perturbation theory, as the leading-order coefficient functions are only expanded up to $\mathcal{O}\left(\alpha_{s}^{0}\right)$ and scaling violations in $\eta_{\bar{q}}\left(Q^{2}\right)$ arise only from the splitting functions at next-toleading order.

The polarized gluon distribution enters $g_{1}\left(x, Q^{2}\right)$ at next-to-leading order. It is only very weakly constrained so far, as no experimental data are available on gluon-initiated processes such as direct- $\gamma$ or heavy meson production. The polarized gluon distribution $\Delta G\left(x, Q^{2}\right)$ is therefore not well-determined by a fit to the $g_{1}$ data alone, and so additional theoretical constraints have to be applied. It follows from the structure of the polarized splitting functions that the small- $x$ behaviour of the gluon and sea quark distributions are closely correlated, which justifies the assumption $a_{G}=a_{\bar{q}}$ in (9). In the region $x>0.1$, structure functions and their evolution are dominated by valence quark contributions, and the impact of the gluon is completely negligible. In Ref. [1] we explored various possibilities for the form of $\Delta G\left(x, Q^{2}\right)$ at large $x$ : hard and soft distributions with the spin aligned with that of the parent hadron, and a distribution with the spin anti-aligned. All three choices give equally good descriptions of the structure function data, but would be relatively easy to discriminate if data on polarized gluon-initiated processes were available. We adopt the same procedure here, i.e. we consider three equally possible scenarios for the behaviour of $\Delta G\left(x, Q_{0}^{2}\right)$, which can be parametrized as follows:

$$
\text { Gluon A : } \quad \gamma_{G}=0, \quad \rho_{G}=0,
$$




$$
\begin{array}{lll}
\text { Gluon B : } & \gamma_{G}=-1, & \rho_{G}=2, \\
\text { Gluon C : } & \gamma_{G}=0, & \rho_{G}=-3 .
\end{array}
$$

Due to the introduction of the additional parameter $\rho_{G}$ in the starting parametrizations (9), these distributions look slightly different from the ones presented in [1].

The normalization $\eta_{G}$ of the gluon distribution can only be determined consistently from the experimental data in a next-to-leading order analysis, where it still has a large error. At leading order, we can estimate $\eta_{G}$ by attributing all the violation of the EllisJaffe sum rule to a large gluon polarization and vanishing sea quark polarization. In this way we obtain $\eta_{G}=1.9$, only slightly different from the value 1.971 obtained in Ref. [1]. Note, however, that at leading order the apportioning of the singlet contribution to $\Gamma_{1}$ between gluons and sea quarks is completely arbitrary [19]. In fact we shall see below that a consistent NLO treatment gives a value of $\eta_{G}$ somewhat less than our estimated leading order value, and similar to the range of values found in Ref. 20.

If parton distributions are interpreted in the probabilistic picture of the naive parton model, the magnitude of the polarized distributions cannot exceed the unpolarized distributions, in order to guarantee positive probabilities for the individual polarization states, i.e.

$$
|\Delta f(x)|<f(x), \quad(f=q, G) .
$$

This is in fact only a rigid constraint at leading order, since parton distibutions at higher orders are only scheme-dependent constants of renormalization and not strict probability densities. The fundamental constraint at arbitrary orders in perturbation theory is the positivity of physical cross sections for all possible helicity configurations, which does not necessarily imply the positivity of the distributions.

Positivity of the polarized distributions is achieved by constraining the parameters of the starting distributions at $Q_{0}^{2}$ such that

$$
\left|\Delta f\left(x, Q_{0}^{2}\right)\right|<f\left(x, Q_{0}^{2}\right), \quad(f=q, G)
$$

Perturbative evolution preserves the positivity of the individual helicity distributions, hence (14) is fulfilled at any $Q^{2}$.

In our leading-order analysis, we use the unpolarized distributions from 16] for reference. At $Q_{0}^{2}=4 \mathrm{GeV}^{2}$ these are

$$
\begin{aligned}
x u_{v}\left(x, Q_{0}^{2}\right)= & 3.221 x^{0.564}(1-x)^{3.726}\left(1-0.6889 x^{0.200}+2.254 x+1.261 x^{3 / 2}\right) \\
x d_{v}\left(x, Q_{0}^{2}\right)= & 0.507 x^{0.376}(1-x)^{4.476}\left(1+1.615 x^{0.553}+3.651 x+1.299 x^{3 / 2}\right) \\
x(\bar{u}+\bar{d})\left(x, Q_{0}^{2}\right)= & {\left[x^{0.158}\left(0.738-0.981 x+1.063 x^{2}\right)(-\ln x)^{0.037}+\right.} \\
& 0.00285 \exp (\sqrt{-4.010 \ln x})](1-x)^{6.356} \\
x s\left(x, Q_{0}^{2}\right)= & 0.0034(-\ln x)^{-1.15}\left(1-2.392 x^{1 / 2}+7.094 x\right)(1-x)^{6.166}
\end{aligned}
$$




$$
\begin{aligned}
& \exp (\sqrt{-6.719 \ln x}) \\
x G\left(x, Q_{0}^{2}\right)= & {\left[x^{0.731}\left(5.110-1.204 x-1.911 x^{2}\right)(-\ln x)^{-0.4718}+\right.} \\
& 0.0527 \exp (\sqrt{-4.584 \ln x})](1-x)^{5.566}
\end{aligned}
$$

The reference unpolarized distributions at next-to-leading order are the $\mathrm{A}^{\prime}$ set of [7], which are parametrized at $Q_{0}^{2}=4 \mathrm{GeV}^{2}$ as

$$
\begin{aligned}
x u_{v}\left(x, Q_{0}^{2}\right) & =2.26 x^{0.559}(1-x)^{3.96}\left(1-0.54 x^{1 / 2}+4.65 x\right) \\
x d_{v}\left(x, Q_{0}^{2}\right) & =0.279 x^{0.335}(1-x)^{4.46}\left(1+6.80 x^{1 / 2}+1.93 x\right) \\
x \operatorname{Sea}\left(x, Q_{0}^{2}\right) & =0.956 x^{-0.17}(1-x)^{9.63}\left(1-2.55 x^{1 / 2}+11.2 x\right) \\
x G\left(x, Q_{0}^{2}\right) & =1.94 x^{-0.17}(1-x)^{5.33}\left(1-1.90 x^{1 / 2}+4.07 x\right)
\end{aligned}
$$

Note that the choice of unpolarized distributions is not crucial for the present analysis. All the widely available leading and next-to-leading order distributions provide very good fits to the unpolarized structure function data, and the small differences between them are much smaller than the precision with which the polarized distributions are currently determined. The flavour decomposition of the unpolarized sea quark distributions is also unimportant for our present analysis and will therefore be disregarded. The starting sea quark distribution of Ref. [7] contains a very small charm quark contribution which can safely be ignored in the present analysis. To be consistent with the evolution of the unpolarized distributions, we take

$$
\Lambda_{\mathrm{LO}}^{n_{f}=4}=200 \mathrm{MeV}[16], \quad \Lambda_{\mathrm{NLO}}^{n_{f}=4}=231 \mathrm{MeV} \text { [7]. }
$$

These correspond to $\alpha_{s}\left(M_{Z}^{2}\right)=0.123(\mathrm{LO})$ and $\alpha_{s}\left(M_{Z}^{2}\right)=0.112(\mathrm{NLO})$.

The parameters most affected by the positivity constraints are the large- $x$ exponents $b_{f}$. For the valence quarks, we fix $b_{u}=b_{u}$ (unpol.) and $b_{d}=b_{u}$ (unpol.) +1 , motivated by counting rule estimates [23]. The parameters $b_{G}$ and $b_{\bar{q}}$ are constrained to be at least as large as their unpolarized counterparts in the fit, but this constraint has only minimal impact. We find that only the $\Delta d_{v}(x)$ distribution tends to saturate positivity, requiring the combination $\gamma_{d}+\rho_{d}$ to be limited in the fit.

The data currently available on $g_{1}$ are not able to test the various theoretical model predictions for the small- $x$ behaviour of the polarized parton distributions [24], which are only expected to apply at much lower values of $x$ [25]. The parameters $a_{f}$ are therefore only effective exponents valid over some finite interval in $x$. It therefore makes no sense to postulate positivity for $x \rightarrow 0$ by constraining the $a_{f}$.

The contribution of charmed quarks to $g_{1}\left(x, Q^{2}\right)$ is negligible at present experimental energies [26] and will not be considered in this analysis. We therefore adopt the evolution procedure of Ref. [16] and fix the number of flavours in the splitting functions at $n_{f}=3$, while the number of flavours in $\alpha_{s}$ increases at each mass threshold,

$$
m_{c}=1.5 \mathrm{GeV}, \quad m_{b}=4.5 \mathrm{GeV}, \quad m_{t}=180 \mathrm{GeV},
$$




\begin{tabular}{|c|r|r|}
\hline & LO & NLO \\
\hline$\eta_{u}$ & 0.823 & 0.918 \\
\hline$\eta_{d}$ & -0.303 & -0.339 \\
\hline$\eta_{G}$ & 1.9 & \\
\hline$\eta_{\bar{q}}$ & -0.0495 & -0.060 \\
\hline$b_{u}$ & 3.73 & 3.96 \\
\hline$b_{d}$ & 4.73 & 4.96 \\
\hline
\end{tabular}

Table 1: Fixed parameters in the $\mathrm{LO}$ and $\mathrm{NLO}(\overline{\mathrm{MS}})$ polarized parton distribution fits.

and $\Lambda\left(n_{f}\right)$ is determined by requiring $\alpha_{s}$ to be continuous across each threshold.

Rather than measuring $g_{1}\left(x, Q^{2}\right)$ directly from absolute cross section differences, it is the relative asymmetry

$$
A_{1}\left(x, Q^{2}\right) \simeq \frac{g_{1}\left(x, Q^{2}\right)}{F_{1}\left(x, Q^{2}\right)}
$$

which is determined experimentally. The structure function $g_{1}\left(x, Q^{2}\right)$ is then inferred using a particular parametrization of $F_{1}\left(x, Q^{2}\right)$. Some experimental groups assume $Q^{2}-$ scaling of $A_{1}\left(x, Q^{2}\right)$ in their extraction of $g_{1}\left(x, Q^{2}\right)$. In order to have a consistent set of data, we have instead used the measured values of $A_{1}\left(x, Q^{2}\right)$ quoted by the experiments and re-evaluated $g_{1}\left(x, Q^{2}\right)$ from Eq. (20)), constructing $F_{1}\left(x, Q^{2}\right)$ from the parametrizations of $F_{2}$ [27] and $R$ [28] which were used in the most recent measurements.

Applying the constraints outlined above, we have used all available world data on $A_{1}^{p, d, n}\left(x, Q^{2}\right)$ [2, 3, 11] to fit the polarized quark and gluon distributions with the parametric forms of (9) imposed at $Q_{0}^{2}=4 \mathrm{GeV}^{2}$. About $35 \%$ of these data were taken at $Q^{2}<Q_{0}^{2}$. To have sensible constraints on the distributions, in particular for $x<0.02$, we include these datapoints in the global fit. The distributions in the region $1 \mathrm{GeV}^{2}<Q^{2}<Q_{0}^{2}$ are obtained by inverting the evolution matrix, which is straightforward in $\mathrm{N}$-moment space.

A problem with using low $Q^{2}$ data points in the fit is the possible contamination by higher-twist contributions. We have tried to estimate to magnitude of such contributions to $g_{1}$ using the parametrization of $F_{2}^{\mathrm{HT}}$ from Ref. [29] and assuming $g_{1}\left(x, Q^{2}\right)^{\mathrm{HT}} \approx$ $g_{1}\left(x, Q^{2}\right)^{\mathrm{LT}}\left(1+C^{\mathrm{HT}}(x) / Q^{2}\right)$. The higher-twist contributions estimated in this way are found to be small for all data-points apart from the two lowest $x$ bins of the SMC experiment.

The global fit is performed using GLAP evolution algorithms in $N$-moment space 30]. The distributions and structure functions are then restored by a numerical inversion into 
$x$ space. The results of the global fit using the leading and next-to-leading order $(\overline{\mathrm{MS}})$ expressions for the splitting functions and the $g_{1}$ coefficient functions are listed in Table 2 . The resulting distributions at $Q_{0}^{2}$ are shown in Figs. 1 (LO) and 2 (NLO).

\begin{tabular}{|l|r|r|r|r|r|r|}
\hline & $\mathrm{A}(\mathrm{LO})$ & $\mathrm{B}(\mathrm{LO})$ & $\mathrm{C}(\mathrm{LO})$ & $\mathrm{A}(\mathrm{NLO})$ & $\mathrm{B}(\mathrm{NLO})$ & $\mathrm{C}(\mathrm{NLO})$ \\
\hline$a_{u}$ & 0.578 & 0.585 & 0.582 & 0.512 & 0.504 & 0.471 \\
\hline$\gamma_{u}$ & 9.38 & 9.31 & 9.50 & 11.65 & 11.98 & 13.14 \\
\hline$\rho_{u}$ & -4.26 & -4.28 & -4.28 & -4.60 & -4.61 & -4.90 \\
\hline$a_{d}$ & 0.666 & 0.662 & 0.660 & 0.780 & 0.777 & 0.809 \\
\hline$\gamma_{d}$ & 10.46 & 10.91 & 11.04 & 7.81 & 8.18 & 6.73 \\
\hline$\rho_{d}$ & -5.10 & -5.09 & -5.06 & -3.48 & -3.61 & -1.99 \\
\hline$\eta_{G}$ & & & & 1.71 & 1.63 & 1.02 \\
\hline$a_{G}$ & 0.520 & 0.524 & 0.456 & 0.724 & 0.670 & 0.425 \\
\hline$b_{G}$ & 9.45 & 6.87 & 8.72 & 5.71 & 5.34 & 11.05 \\
\hline$b_{\bar{q}}$ & 15.06 & 15.96 & 11.82 & 14.40 & 18.06 & 16.40 \\
\hline$\gamma_{\bar{q}}$ & 2.30 & 2.42 & 2.11 & 4.63 & 5.30 & -2.67 \\
\hline$\rho_{\bar{q}}$ & -2.00 & -2.00 & -1.95 & -4.96 & -5.25 & -3.08 \\
\hline \hline$\chi^{2}$ & 98.3 & 97.7 & 100.0 & 89.7 & 91.0 & 93.4 \\
\hline & & & & & & \\
\hline
\end{tabular}

Table 2: Fitted parameters in the $\mathrm{LO}$ and $\mathrm{NLO}(\overline{\mathrm{MS}})$ polarized parton distributions at $Q_{0}^{2}$. The $\chi^{2}$ values are with respect to the 110 data points included in the global fit.

The resulting parameters are not independent of each other. In particular, $a_{u}, a_{d}$ and $a_{G}=a_{\bar{q}}$ are strongly correlated. The $a_{i}$ of the valence distributions are anticorrelated with the corresponding $\gamma_{i}$, reflecting the fact that $a_{i}$ is only an effective exponent for a finite range in $x$. The $\gamma_{i}$ and $\rho_{i}$ are also anticorrelated. The $\chi^{2}$ distribution is very flat around the local minima found by the global fits, especially with respect to the gluon and sea quark parameters.

The three gluon scenarios give fits of almost identical quality, reflecting the small impact of the gluon distribution on $g_{1}\left(x, Q^{2}\right)$ at large and medium $x$. The $\chi^{2}$ obtained in the NLO fits are systematically lower due to the additional degree of freedom given by the normalization of the polarized gluon distribution. All fits give very good descriptions 
for the polarized structure functions $g_{1}^{p, n, d}\left(x, Q^{2}\right)$. This is illustrated in Fig. 3, which shows the NLO description of the various $g_{1}$ measurements using Gluon A. The curves correspond to $Q^{2}=1,4,10,50 \mathrm{GeV}^{2}$, reflecting the spread in $Q^{2}$ values of the different data sets. There is a systematic decrease in the $Q^{2}$ values of the data points from large $x$ to small $x$.

The contributions of $u_{v}\left(x, Q^{2}\right)$ and $d_{v}\left(x, Q^{2}\right)$ to the neutron structure function $g_{1}^{n}\left(x, Q^{2}\right)$ are almost equal in magnitude but opposite in sign. The neutron structure function is therefore much more sensitive to the sea quark polarization than $g_{1}^{p}\left(x, Q^{2}\right)$ and $g_{1}^{d}\left(x, Q^{2}\right)$. It displays a clear double peak structure, as the sea quarks are dominant in a different $x$-region than the valence quarks. A precision measurement of $g_{1}^{n}\left(x, Q^{2}\right)$ [31 will therefore be able to provide vital new information on the shape of the sea quark polarization.

From a consideration of the size of the errors on the various fitted parameters, it is apparent that the world data on $g_{1}\left(x, Q^{2}\right)$ really only constrain the polarized valence quark distributions and, to a lesser extent, the overall magnitude of the sea quark polarization. The flavour decomposition of the polarized sea is still completely unknown. Only dedicated experiments, such as the production of Drell-Yan lepton pairs or the flavour-tagging of final-state hadrons in polarized deep inelastic scattering, will be able to provide further information. Most important of all, the polarized gluon distribution is almost completely undetermined, as its impact on the polarized structure function is less than the present experimental accuracy. The variation between our three gluon sets certainly underestimates the true uncertainty in the distributions.

\section{Prospects for future measurements}

There are several measurements which could be feasible at future experiments and which yield additional information on the polarized gluon distribution. In this section we present some representative predictions for two of these: the $Q^{2}$ dependence of $g_{1}\left(x, Q^{2}\right)$, and an asymmetry in $J / \psi$-photoproduction. We also briefly discuss the prospects of a measurement of polarized structure functions at the HERA collider.

The derivative of $F_{2}\left(x, Q^{2}\right)$ with respect to $Q^{2}$ has been used to measure the unpolarized gluon distribution in fixed target experiments [32] $(0.008<x<0.5)$ and at HERA [33] $\left(2 \cdot 10^{-4}<x<3 \cdot 10^{-2}\right)$. The method is particularly powerful at small $x$, where the gluon distribution dominates the $Q^{2}$ evolution, $\partial F_{2}(x) / \partial \ln Q^{2} \sim P_{q G}(y) \otimes G(x / y)$. In the same way, we can use our three sets of distributions $\mathrm{A}, \mathrm{B}$ and $\mathrm{C}$ to explore the sensitivity of the polarized structure function evolution to $\Delta G\left(x, Q^{2}\right)$. Figure 4 shows the predictions for the asymmetry $A_{1}\left(x, Q^{2}\right)$ as a function of $Q^{2}$ in the kinematic range representative of current fixed-target experiments. The asymmetry is obtained from Eq. (20) with $g_{1}$ calculated using our polarized distributions and $F_{1}$ calculated using the NLO unpolarized $\operatorname{MRS}\left(\mathrm{A}^{\prime}\right)$ distributions of Ref. [7]. The latter are extrapolated to lower values of 
$Q^{2}$, which reproduces the full backwards evolution to within a few per cent. At large $x$, there is no sensitivity to $\Delta G\left(x, Q^{2}\right)$ - the evolution is completely dominated by the quark contribution. At small $x$, on the other hand, we see some dependence on the gluon. In this region $\Delta P_{q G}(y) \otimes \Delta G(x / y)<0$, and so the derivative $\partial A_{1} / \partial \ln Q^{2}$ is more negative for the sets that have a larger gluon polarization above the $x$ value considered. In particular, we see that at $x \sim 0.01$ the proton asymmetry is almost $Q^{2}$ independent for Set C, but decreases with increasing $Q^{2}$ for Sets A and B. Unfortunately, the sensitivity of the present experiments is much worse than the differences between the various sets. To illustrate this, we have included two data points at $x=0.035$ from the recent SMC and E143 measurements. For the deuteron, the quark contribution to the structure function is smaller, and so the dependence on $\Delta G\left(x, Q^{2}\right)$ at small $x$ is somewhat enhanced. Considering the large errors on the present data, it seems doubtful that a measurement of the polarized gluon distribution from the $Q^{2}$ variation of $A_{1}\left(x, Q^{2}\right)$ is feasible for values of $Q^{2}$ where perturbative expressions can be safely applied. Future high statistics experiments at higher beam energies [34 will clearly improve this measurement. It still has to be kept in mind that a determination of $\Delta G\left(x, Q^{2}\right)$ from the $Q^{2}$ dependence of $A_{1}\left(x, Q^{2}\right)$ can never reach the quality of the corresponding unpolarized measurement, as the gluonic contribution is not as dominant in the evolution of polarized parton distributions as it is in the unpolarized evolution [25].

Inelastic $J / \psi$ production from a nucleon target directly probes the gluon distribution via the photon-gluon fusion subprocess $\gamma^{\star}+g \rightarrow(c \bar{c})+g$, which can be described in the colour-singlet model of Ref. [35]. Depending on the virtuality of the photon, one can distinguish two different classes of events: photoproduction $\left(Q^{2} \approx 0\right)$ and leptoproduction $\left(Q^{2} \gg 0\right)$. The EMC [36] and NMC experiments [37 have obtained measurements of the unpolarized leading-order gluon distribution in the range $x \sim 0.05-0.25$ from this process. The results agree well with gluon distributions extracted from other processes, for example large $-p_{T}$ direct photon production. The corresponding cross section for polarized $J / \psi$ leptoproduction has been calculated in Ref. [38]. Taking the photoproduction limit $Q^{2} \rightarrow 0$, one obtains

$$
\begin{aligned}
\frac{\mathrm{d} \sigma_{\lambda h}^{\gamma N}\left(E_{\gamma}\right)}{\mathrm{d} p_{T}^{2} \mathrm{~d} z}= & \frac{2 \pi}{\alpha} \cdot \eta G_{\lambda}\left(\eta, M_{J / \psi}^{2}\right) \cdot \frac{8 \alpha_{s}^{2} M_{J / \psi} \Gamma_{J / \psi \rightarrow e^{+} e^{-}}}{3} \cdot \frac{z(1-z)}{\left[M_{J / \psi}^{2}(1-z)+p_{T}^{2}\right]^{2}} \\
& \times[A(z)+h \lambda C(z)] \cdot \mathcal{F}\left(p_{T}^{2}, z\right)
\end{aligned}
$$

with

$$
\begin{aligned}
\mathcal{F}\left(p_{T}^{2}, z\right) & =\frac{z^{2}(1-z)^{2}}{\left(p_{T}^{2}+(1-z)^{2} M_{J / \psi}^{2}\right)^{2}} \frac{1}{\left(p_{T}^{2}+M_{J / \psi}^{2}\right)^{2}} \\
A(z) & =\frac{M_{J / \psi}^{2}}{2}\left(z^{2}\left(M_{J / \psi}^{2}-z s_{\gamma N}\right)^{2}+(1-z)^{2}\left(M_{J / \psi}^{2}+\right.\right.
\end{aligned}
$$




$$
\begin{aligned}
& \left.\left.(1-z) s_{\gamma N}\right)^{2}+\left(s_{\gamma N}-M_{J / \psi}^{2}\right)^{2}\right) \\
C(z)= & (1-z)\left(M_{J / \psi}^{2}-z s_{\gamma N}\right)\left(z M_{J / \psi}^{2}\left(M_{J / \psi}^{2}-z s_{\gamma N}\right)+(z-2) M_{J / \psi}^{2} s_{\gamma N}\right) \\
s_{\gamma N}= & 2 M_{N} E_{\gamma} \\
\eta= & \frac{p_{T}^{2}+(1-z) M_{J / \psi}^{2}}{z(1-z) s_{\gamma N}} \\
z= & \frac{E_{J / \psi}}{E_{\gamma}} \\
\lambda= & \text { gluon helicity } \\
h= & \text { lepton helicity }
\end{aligned}
$$

The polarized (unpolarized) cross sections are obtained by taking the difference (sum) over the helicity states. The polarized cross section is then proportional to $\Delta G(\eta)=G_{+}(\eta)-$ $G_{-}(\eta)$ and depends only on $C(z)$, while the unpolarized cross section is proportional to $G(\eta)=G_{+}(\eta)+G_{-}(\eta)$ and depends only on $A(z)$. Taking the cross sections integrated over one of the variables, one can define two physics asymmetries:

$$
\begin{aligned}
\mathcal{A}(z) & =\frac{\mathrm{d} \Delta \sigma^{\gamma N}\left(E_{\gamma}\right)}{\mathrm{d} z} / \frac{\mathrm{d} \sigma^{\gamma N}\left(E_{\gamma}\right)}{\mathrm{d} z}, \\
\mathcal{A}\left(p_{T}^{2}\right) & =\frac{\mathrm{d} \Delta \sigma^{\gamma N}\left(E_{\gamma}\right)}{\mathrm{d} p_{T}^{2}} / \frac{\mathrm{d} \sigma^{\gamma N}\left(E_{\gamma}\right)}{\mathrm{d} p_{T}^{2}} .
\end{aligned}
$$

With a photon beam of energy $E_{\gamma}=45 \mathrm{GeV}$ and cuts on $p_{T}^{2}$ and $z\left(p_{T}^{2}>p_{T \text { min }}^{2}=\right.$ $0.25 \mathrm{GeV}^{2}, z<z_{\max }=0.9$, as proposed in [39]), one gets significantly different asymmetries for the three gluon distributions $\mathrm{A}, \mathrm{B}$ and C. Predictions for the asymmetry $\mathcal{A}(z)$, calculated using the three leading order distributions in combination with the unpolarized leading order gluon distributions of Ref. [16], are shown in Fig. 5. Note that small z corresponds to large $\eta$, and the ordering of the asymmetry in this region simply reflects the ordering of the different $\Delta G(\eta)$ at large $\eta$. Due to the relatively large asymmetry of up to $15 \%$, such a measurement could provide vital information on the leading order polarized gluon distribution for $0.1<x<0.35$ and $Q^{2} \approx M_{J / \psi}^{2}$.

In the foreseeable future, the HERA collider may be able to accelerate polarized protons [40]. This would offer the unique opportunity of measuring the polarized structure function $g_{1}^{p}\left(x, Q^{2}\right)$ far beyond the $Q^{2}$ range of present fixed target experiments. In order to judge the quality of the new information gained from such a measurement, an orderof-magnitude estimate of the statistical errors is crucial [41. The structure function $g_{1}^{p}\left(x, Q^{2}\right)$ is extracted from a measurement of the asymmetry

$$
A_{\|}\left(x, Q^{2}\right)=\lambda_{e} \lambda_{p} D(y) \frac{g_{1}^{p}\left(x, Q^{2}\right)}{F_{1}^{p}\left(x, Q^{2}\right)},
$$


where $\lambda_{e}\left(\lambda_{p}\right)$ denote the polarizations of the electron (proton) beam and

$$
D=\frac{2 y-y^{2}}{2(1-y)\left(1+R^{p}\right)+y^{2}}
$$

is the kinematical depolarization of the photon. The statistical error on $x g_{1}\left(x, Q^{2}\right)$ is therefore 41]:

$$
\begin{aligned}
\delta\left[x g_{1}^{p}\left(x, Q^{2}\right)\right] & =\frac{1}{2 \lambda_{e} \lambda_{p}} \frac{1+(1-y)^{2}}{1-(1-y)^{2}}\left[2 x F_{1}^{p}\left(x, Q^{2}\right)+\frac{2(1-y)}{1+(1-y)^{2}} F_{L}^{p}\left(x, Q^{2}\right)\right] \\
& \times\left(\mathcal{L}_{\text {int }} \int\left[\mathrm{d}^{2} \sigma^{\text {(unpol. })} /\left(\mathrm{d} x \mathrm{~d} Q^{2}\right)\right] \mathrm{d} x \mathrm{~d} Q^{2}\right)^{-1 / 2} \sqrt{1-A_{\|}\left(x, Q^{2}\right)}
\end{aligned}
$$

where the unpolarized differential cross section is integrated over the bin used in the measurement.

To study the accuracy of a measurement of $g_{1}^{p}\left(x, Q^{2}\right)$ in the collider mode of HERA, we have evaluated the above expression using the unpolarized parton distribution set $\operatorname{MRS}\left(\mathrm{A}^{\prime}\right)$ from Ref. [7] with the next-to-leading order polarized parton distribution set A described in Section 2. We apply the following cuts to the HERA phase space [42]: $0.1<y<0.95, Q^{2}>1 \mathrm{GeV}^{2}, \Theta_{e^{\prime}}<176^{\circ}$ and $E_{e^{\prime}}>5 \mathrm{GeV}$, and consider two scenarios for the beam energies:

$$
\begin{aligned}
& \text { (a) }: \sqrt{s}=300 \mathrm{GeV} \quad \text { with } \quad E_{e}=27.44 \mathrm{GeV}, E_{p}=820 \mathrm{GeV} \\
& \text { (b) }: \sqrt{s}=150 \mathrm{GeV} \quad \text { with } \quad E_{e}=18.75 \mathrm{GeV}, E_{p}=300 \mathrm{GeV}
\end{aligned}
$$

The expected errors for an integrated luminosity of $60 \mathrm{pb}^{-1}$ at $\lambda_{e}=\lambda_{p}=0.8$ in these two scenarios are shown in Fig. 6. We take two bins per decade in $x$ and only one bin in $Q^{2}$. These results are consistent with the leading-order estimates of [41], bearing in mind the different cuts applied. In particular, we estimate smaller errors in the small- $x$ region, as we apply no cuts on the hadroinc final state.

The average $Q^{2}$ values probed at HERA range from $2 \mathrm{GeV}^{2}\left(x \approx 6 \cdot 10^{-5}\right)$ to $1060 \mathrm{GeV}^{2}$ $(x \approx 0.05)$ for $\sqrt{s}=300 \mathrm{GeV}$ and from $1.7 \mathrm{GeV}^{2}\left(x \approx 2 \cdot 10^{-4}\right)$ to $270 \mathrm{GeV}^{2}(x \approx 0.05)$ for $\sqrt{s}=150 \mathrm{GeV}$. For reference, we also show in Fig. 6 a parametrization of $g_{1}^{p}\left(x, Q^{2}\right)$ obtained from the NLO set A. To illustrate the impact of a measurement at HERA, we include the three lowest datapoints reported by the SMC experiment [1], corresponding to $Q^{2}$ values around $1.5 \mathrm{GeV}^{2}$. It is apparent that a measurement at lower beam energies will yield data of higher statistical quality. In contrast, the higher beam energies yield a measurement at smaller $x$.

A measurement of $g_{1}^{p}\left(x, Q^{2}\right)$ at the HERA collider will evidently not provide a large number of precision data on the $x$ and $Q^{2}$ dependences of this structure function. Hence, it will not provide sufficient information for an indirect determination of the polarized 
gluon density. The important physics result of such a measurement is the determination of the small- $x$ behaviour of $g_{1}^{p}\left(x, Q^{2}\right)$, for which various, significantly different predictions exist [24]. It is important to stress that a measurement of $g_{1}^{p}\left(x, Q^{2}\right)$ at small $x$ will reduce the experimental uncertainty on the Ellis-Jaffe sum rule. The impact of the small $x$ region can be easily seen in Fig. 6, as the Ellis-Jaffe sum rule is proportional to the area enclosed by $x g_{1}^{p}\left(x, Q^{2}\right)$ and the $x$ axis.

A final remarkable point on the measurability of $g_{1}^{p}\left(x, Q^{2}\right)$ at HERA is the impact on the statistical error of the minimum cut on $y$. As the photon depolarizes for small $y$, a small cut on $y$ (such as $y>0.01$ ) diminishes the statistical accuracy, even though more data are included in the bin. We find that a minimal cut on $y$ between 0.1 and 0.2 yields the optimal accuracy.

\section{Summary and Conclusions}

We have performed leading and next-to-leading order QCD fits to the world data on the $g_{1}$ polarized structure function measured with proton, neutron and deuteron targets. The quality of the fits is excellent, and from them we obtain sets of polarized parton distributions which can be used for further phenomenological analyses. The experimental precision is highest for the proton and deuteron data, and together these constrain the shapes of the valence $u$ and $d$ distributions. The sea quark and gluon distributions are still largely undetermined. There is a weak constraint on the overall size of the former, but almost no information at all on the flavour decomposition of the sea. Following our earlier work, we have presented three qualitatively different gluon distributions, characterized by different behaviours at large $x$.

It seems that further progress in the determination of polarized parton distributions will require measurements other than $g_{1}$ at fixed-target experiments. Inelastic $J / \psi$ photoproduction in lepton-hadron scattering has already proved capable of measuring the unpolarized gluon distribution, and a similar measurement with a polarized beam and target would provide the first real information on $\Delta G\left(x, Q^{2}\right)$. A measurement of the $Q^{2}$ dependence of $A_{1}\left(x, Q^{2}\right)$ can help to estimate the overall amount of gluon polarization at higher and medium $x$. Althogh present experiments cannot provide such a measurement due to low statistics or too low values of $Q^{2}$, first information can be expected from the next generation of fixed target experiments. We have also studied the accuracy of a measurement of $g_{1}\left(x, Q^{2}\right)$ at the HERA collider with polarized beams. Such a measurement will help to discriminate between various predictions for the behaviour of $g_{1}\left(x, Q^{2}\right)$ at small $x$, and will reduce the uncertainty on the Ellis-Jaffe sum rule from the extrapolation to small $x$.

\footnotetext{
${ }^{2}$ The FORTRAN code for the various sets described in this paper is available by electronic mail from T.K.Gehrmann@durham.ac.uk
} 


\section{Acknowledgements}

Financial support from the UK PPARC (WJS), and from the Gottlieb Daimler- und Karl Benz-Stiftung and the Studienstiftung des deutschen Volkes (TG) is gratefully acknowledged. This work was supported in part by the EU Programme "Human Capital and Mobility", Network "Physics at High Energy Colliders", contract CHRX-CT93-0357 (DG

12 COMA). We would like to thankl Alan Martin, Werner Vogelsang and Dick Roberts for useful discussions.

\section{References}

[1] T. Gehrmann and W.J. Stirling, Z. Phys. C65 (1995) 461.

[2] SMC collaboration: D. Adams et al., Phys. Lett. B357 (1995) 248.

[3] SLAC-E143 collaboration: K. Abe et al., Phys. Rev. Lett. 74 (1995) 346; 75 (1995) 25.

[4] SLAC-E143 collaboration: K. Abe et al., SLAC-PUB-95-6982 (1995).

[5] R. Mertig and W.L. van Neerven, Leiden preprint INLO-PUB-6/95 (revised) (1995). W. Vogelsang, Rutherford Laboratory preprint RAL-TR-95-071 (1995).

[6] M. Glück, E. Reya and W. Vogelsang, Phys. Lett. B359 (1995) 201.

M. Glück, E. Reya, M. Stratmann and W. Vogelsang, Dortmund preprint DO-TH 95-13 (1995).

[7] A.D. Martin, R.G. Roberts and W.J. Stirling, Phys. Lett. B354 (1995) 155.

[8] CTEQ collaboration: H.L. Lai et al., Phys. Rev. D51 (1995) 4763.

[9] M. Gourdin, Nucl. Phys. B38 (1972) 418.

[10] J. Ellis and R.L. Jaffe, Phys. Rev. D9 (1974) 1444; Erratum D10 (1974) 1669.

[11] SLAC-Yale collaboration: M.J. Alguard et al., Phys. Rev. Lett. 37 (1976) 1261; G. Baum et al., Phys. Rev. Lett. 45 (1980) 2000; 51 (1983) 1135.

EMC collaboration: J. Ashman et al., Nucl. Phys. B328 (1989) 1.

SLAC-E142 collaboration: D.L. Anthony et al., Phys. Rev. Lett. 71 (1993) 959.

SMC collaboration: B. Adeva et al., Phys. Lett. B302 (1993) 553; D. Adams et al., Phys. Lett. B329 (1994) 399. 
[12] M.A. Ahmed and G.G. Ross, Nucl. Phys. B111 (1976) 441.

K. Sasaki, Progr. Theor. Phys. 54 (1975) 1816.

G. Altarelli and G. Parisi, Nucl. Phys. B126 (1977) 298.

[13] J. Kodaira, S. Matsuda, T. Muta, K. Sasaki and T. Uematsu, Phys. Rev. D20 (1979) 627.

[14] J. Kodaira, Nucl. Phys. B165 (1980) 129.

[15] E.G. Floratos, D.A. Ross and C.T. Sachrajda, Nucl. Phys. B129 (1977) 66; Errata B139 (1978) 545, B152 (1979) 493.

A. Gonzales-Arroyo, C. Lopez and F.J. Yndurain, Nucl. Phys. B153 (1979) 161.

G. Curci, W. Furmanski and R. Petronzio, Nucl. Phys. B175 (1980) 27,

W. Furmanski and R. Petronzio, Phys. Lett. B97 (1980) 437.

E.G. Floratos, C. Kounnas and R. Lacaze, Nucl. Phys. B192 (1981) 417.

[16] M. Glück, E. Reya and A. Vogt, Z. Phys. C67 (1995) 433.

[17] M. Anselmino, A. Efremov and E. Leader, Phys. Rep. 261 (1995) 1.

[18] C.S. Lam and Bing-An Li, Phys. Rev. D25 (1982) 683.

[19] E.B. Zijlstra and W.L. van Neerven, Nucl. Phys. B417 (1994) 61; Erratum B426 (1994) 245.

[20] R.D. Ball, S. Forte and G. Ridolfi, preprint CERN-TH/95-266 (1995).

[21] D.A. Ross and C.T. Sachrajda, Nucl. Phys. B149 (1979) 497.

M. Stratmann, W. Vogelsang and A. Weber, Dortmund preprint DO-TH 95-15 (1995).

[22] -266 F.E. Close and R.G. Roberts, Phys. Lett. B316 (1993) 165.

[23] S.J. Brodsky, M. Burkardt and I. Schmidt, Nucl. Phys. B441 (1995) 197.

[24] M.A. Ahmed and G.G. Ross, Phys. Lett. 56B (1975) 385.

F.E. Close and R.G. Roberts, Phys. Lett. B336 (1994) 257.

S.D. Bass and P.V. Landshoff, Phys. Lett. B336 (1994) 537.

R.D. Ball, S. Forte and G. Ridolfi, Nucl. Phys. B444 (1995) 287; Erratum B449 (1995) 680.

J. Bartels, B.I. Ermolaev and M.G. Ryskin, DESY preprint DESY-95-124 (1995).

J. Blümlein and A. Vogt, DESY preprint DESY-95-175 (1995).

J. Kwiecziǹski, Durham preprint DTP/95/98 (1995), to appear in Acta Physica Polonica. 
[25] T. Gehrmann and W.J. Stirling, Durham preprint DTP/95/62 (1995), to appear in Phys. Lett. B.

[26] M. Glück, E. Reya and W. Vogelsang, Nucl. Phys. B351 (1991) 579.

[27] NMC collaboration: P. Amaudruz et al., Phys. Lett. B295 (1992) 159.

[28] L.W. Whitlow et al., Phys. Lett. B282 (1992) 159.

[29] M. Virchaux and A. Milsztajn, Phys. Lett. B274 (1992) 221.

[30] W. Furmanski and R. Petronzio, Z. Phys. C11 (1982) 293.

[31] HERMES collaboration: Technical Design report, DESY (1993).

[32] NMC collaboration: M. Arneodo et al., Phys. Lett. B309 (1993) 222.

[33] H1 collaboration: S. Aid et al., Phys. Lett. B354 (1995) 494. ZEUS collaboration: M. Derrick et al., Phys. Lett. B345 (1995) 576.

[34] SLAC-E154/E155 collaborations: proposals (1994).

[35] E.L. Berger and D. Jones, Phys. Rev. D23 (1981) 1521. R. Baier and R. Rückl, Nucl. Phys. B218 (1983) 289.

[36] EMC collaboration: J. Ashman et al., Z. Phys. C56 (1992) 21.

[37] NMC collaboration: D. Allasia et al., Phys. Lett. B258 (1991) 493.

[38] J.Ph. Guillet, Z. Phys. C39 (1988) 75.

[39] V. Breton, Measurement of $\Delta G$ by $J / \psi$ Photoproduction at SLAC, SLAC proposal, January 1994.

[40] Proceedings of the "Workshop on the Prospects of Spin Physics at HERA, August 28-31, 1995, DESY-Zeuthen, Zeuthen, Germany", eds. J. Blümlein and W.D. Nowak, DESY report, to appear.

[41] J. Blümlein, DESY preprint DESY-95-164.

[42] M. Klein, Proceedings of the workshop "Physics at HERA", Hamburg, October 2930, 1991, eds. W. Buchmüller and G. Ingelman, p. 71. 


\section{Figure Captions}

[1] Leading order polarized parton distributions as described in the text at $Q_{0}^{2}=4 \mathrm{GeV}^{2}$ compared to the unpolarized distributions of [16].

[2] Next-to-leading order polarized parton distributions as described in the text at $Q_{0}^{2}=4 \mathrm{GeV}^{2}$ compared to the unpolarized distributions of $[7]$.

[3] Structure function measurements $x g_{1}\left(x, Q^{2}\right)$ of the proton, deuteron and neutron compared to the next-to-leading order predictions obtained using Gluon A.

[4] $Q^{2}$ dependence of $A_{1}^{p, d}\left(x, Q^{2}\right)$ in next-to-leading order using the Gluons $\mathrm{A}, \mathrm{B}$ and C. To illustrate the sensitivity of current experiments, we show the $x=0.035$ datapoints from the recent SMC and E143 measurements.

[5] Predictions for the asymmetry $A(z)$ (Eq. (22)) in polarized $J / \psi$ photoproduction at $E_{\gamma}=45 \mathrm{GeV}$, using the different leading order polarized gluon distributions.

[6] Expected errors for a measurement of $g_{1}^{p}\left(x, Q^{2}\right)$ at the HERA collider with $\sqrt{s}=$ $300 \mathrm{GeV}$ and $\sqrt{s}=150 \mathrm{GeV}$. 

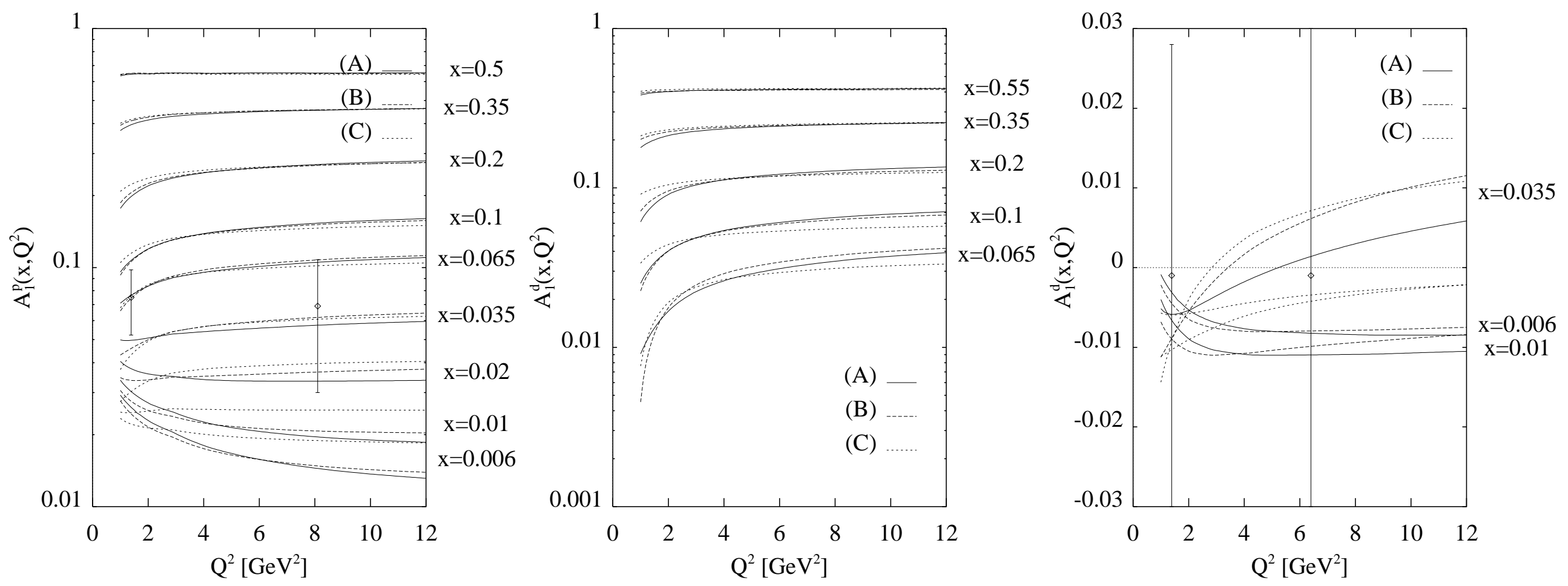

Fig. 4 

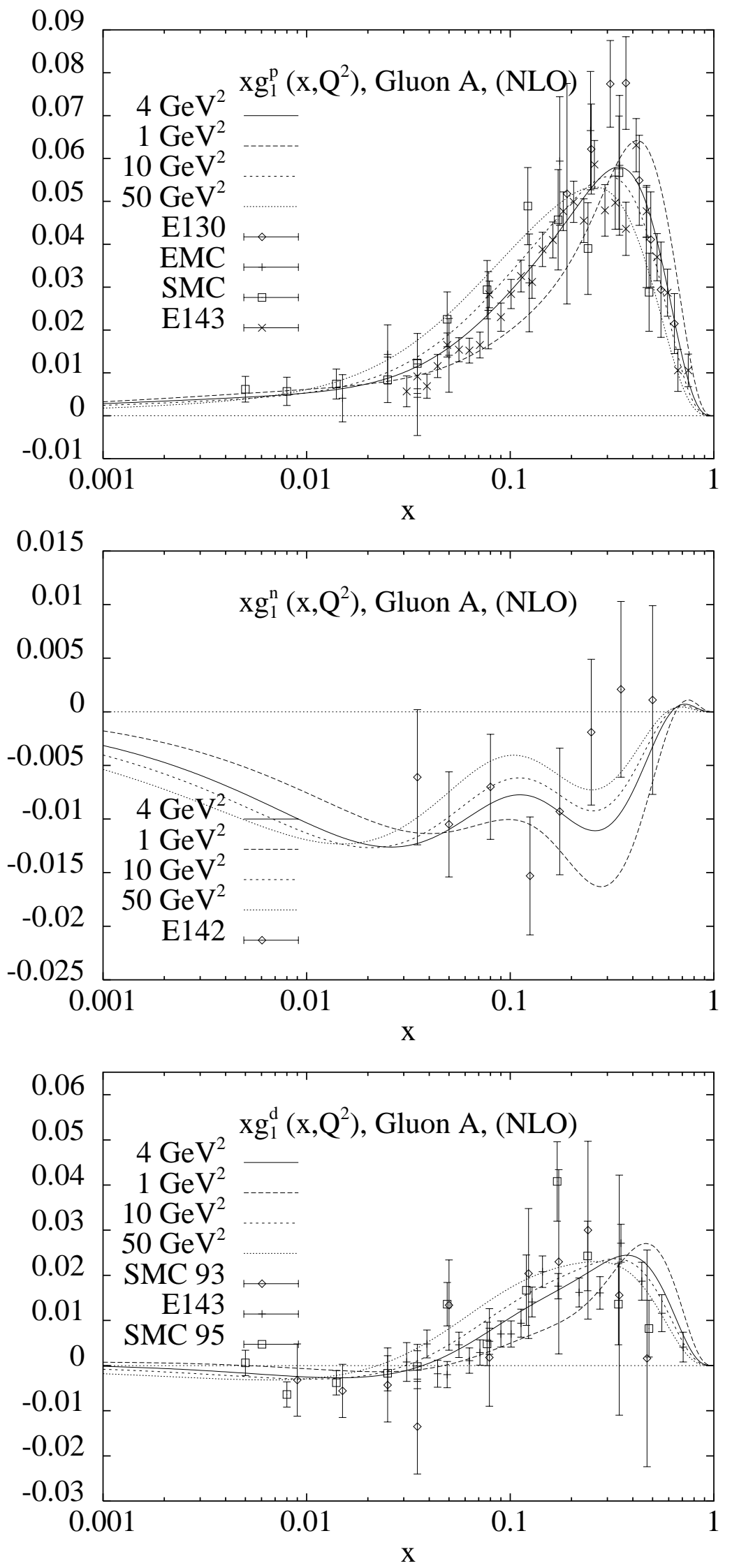

Fig. 3 

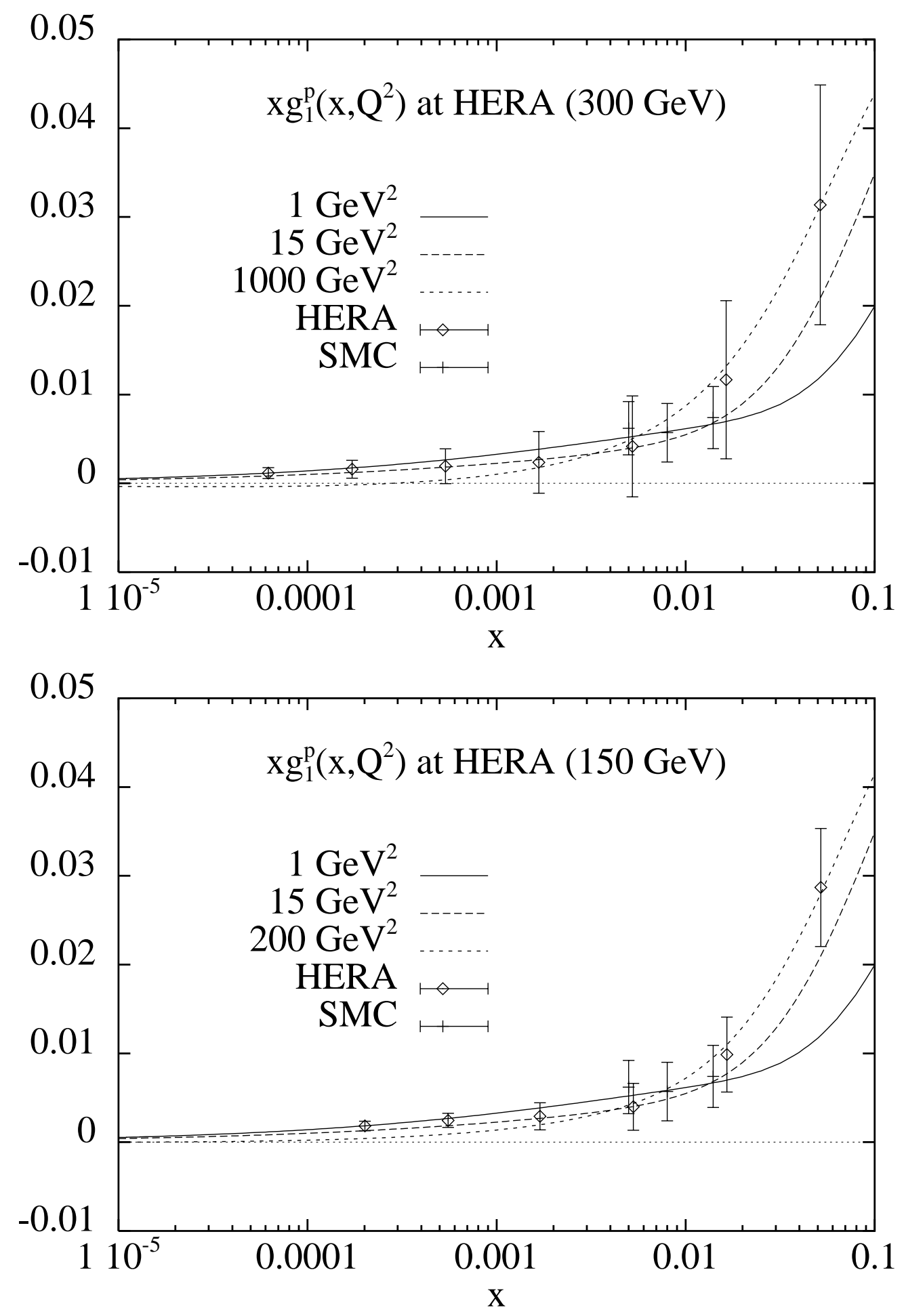

Fig. 6 

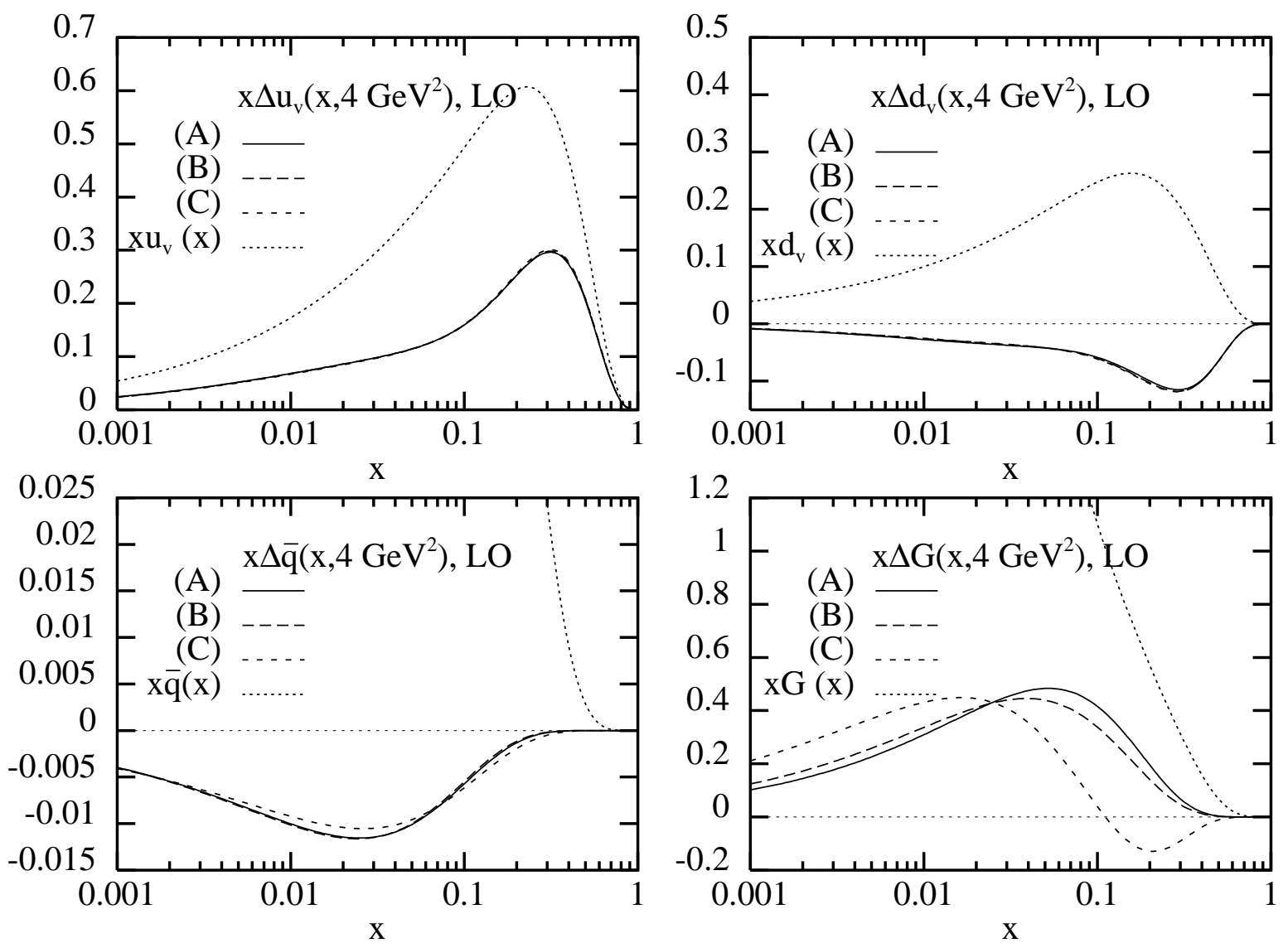

Fig. 1 

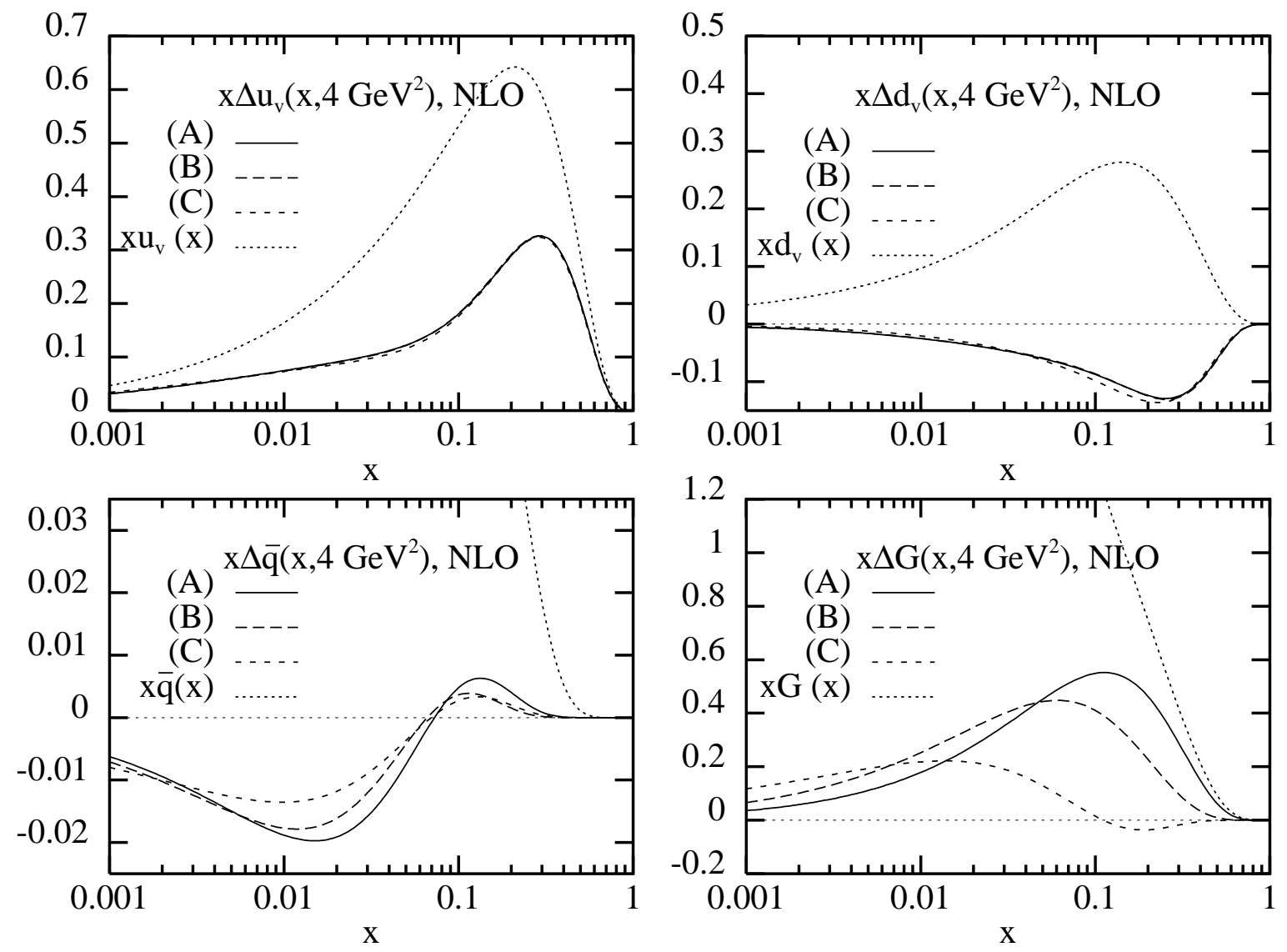

Fig. 2 


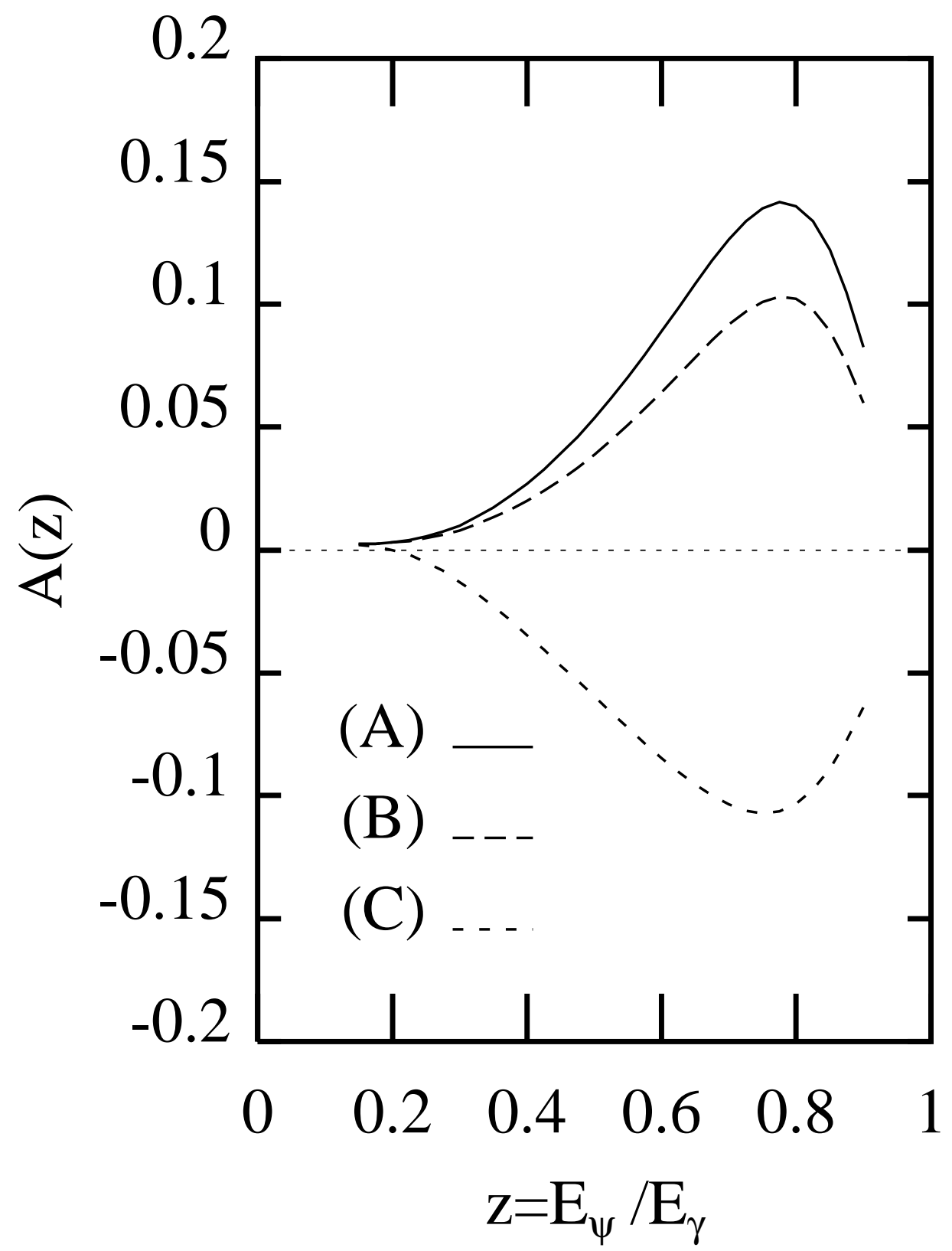

Fig. 5 